\title{
Features of optical properties of ferromagnetic semiconductors with dynamic laser-induced gratings
}

\author{
O.Yu. Semchuk ${ }^{1}$, R.Z. Veskliarskii ${ }^{2}$, and K.G. Kosharskii ${ }^{2}$ \\ ${ }^{1}$ Institute of Surface Chemistry National academy of Sciences of Ukraine, \\ 31 Prospect Nauky \\ ${ }^{2}$ Department of Physics, Kyiv Taras Shevchenko National University, 17 General Naumov Str., 03164, Kyiv, Ukraine \\ 64 Volodimirs'ka Str., Kyiv 03033, Ukraine
}

\begin{abstract}
Nonlinear optical phenomena in ferromagnetic semiconductor with dynamic grating created by coherent light beams are investigated. A high-frequency current and nonlinear absorption coefficient are calculated. It is shown that with increasing wave frequency, the nonlinear absorption coefficient grows and reaches the maximum at frequency $\omega \approx 3 \cdot 10^{12} \mathrm{~s}^{-1}$, and then smoothly decreases at higher frequencies. Therefore, the second term of the nonlinear absorption coefficient is modulated with coordinate. This proves that a dynamic grating emerges in ferromagnetic semiconductor.
\end{abstract}

Keywords: ferromagnetic semiconductor, dynamic grating, electron, magnon, absorption, high-frequency current, wave vector, coherent light beams, vector-potential.

Paper received 20.06.01; revised manuscript received 08.10.01; accepted for publication 12.12.01.

\section{Introduction}

Nowadays rapid development of electronic engineering, holography and semiconductor infrared technology initiates further theoretical and experimental studies of magnetic solids exposed to intensive high-frequency fields of the coherent electromagnetic waves. Ferromagnetic semiconductors (FMSC) are the most interesting materials for this study.

The electron-magnon system of a FMSC acquires new properties in a high-frequency electromagnetic wave field. In particular, the effect of a high-frequency field on the collisions of electrons with magnons and impurities become important at frequencies of coherent electromagnetic waves more than average carrier energy. This leads to a number of new nonlinear and non-equilibrium effects reviewed in detail in [1].

On the other hand, the situation when several coherent light beams (CLB) rather than a single beam are incident on a FMSC is of special interest. Interference effects lead to new features of the interaction of a high-frequency field with free carriers and magnons. Formally, the situation with a single and several CLB is different, since the scattering probability of a carrier on magnons and impurities in a sample illuminated by a single and several CLB is spatially modulated. Besides, the transport equation for electrons contains a spatial modulated force of the high-frequency pressure of the optical field acting on free carriers. Spatial modulation of the collision integral and force caused by the high-frequency pressure on electrons due to the interference effects can generate static and dynamic gratings in the system of non-equilibrium electrons and magnons in a FMSC. The mechanism leading to the formation of such gratings in FMSC was proposed in [2.3].

Currently, the FMSC with static gratings are under intensive investigation. The transport (electroconductivity) and optical (refractive index) phenomena in FMSC with gratings have already been studied. These and other phenomena are described in [4]. In [5] the light reflection coefficient of the FMSC with gratings was calculated, and it was shown that the presence of gratings leads to a modulation of the light absorption coefficient in the FMSC. Voltage-current characteristics of the FMSC with gratings were investigated in [6].

In this work we have made the theoretical investigation of the dynamic grating on non-equilibrium electrons and magnons in FMSC as well as the propagation and absorption of the electromagnetic waves in FMSC in a dynamical regime. The results obtained in the frame of this work may be useful for elaboration of new recording methods, for physics of dynamical holograms, infrared semiconductor techniques, as well as the applications mentioned above. 
O.Yu. Semchuk et al.: Features of optical properties of ferromagnetic ...

\section{System of quantum kinetic equations for electrons and magnons}

A wide-gap FMSC of the EuO type with an average carrier (electron) concentration $n_{0}$ in an external constant electric field $\vec{F}_{0} \| O Z$ in the spin-wave temperature range is considered. Assume several CLB on the outer surface, $x=0$ of the FMSC (Fig.1.), and the vector-potential of the CLB's given by the next expression $\vec{A}(\vec{r}, t)=\sum_{j} \vec{A}_{j} \cos \left(\omega t-\vec{k}_{j} \vec{r}-\varphi_{j}\right)$, and their frequency $\omega$ satisfies the inequality $\bar{\varepsilon}<<\omega<<\varepsilon_{g}$ ( $\bar{\varepsilon}$ is an average carrier energy, $\varepsilon_{g}$ is the gap energy). It follows that CLB does not change the total number of electrons in FMSC that can lead to a redistribution of their density in conduction band. Electrons are considered to be non-generate, and their average energy $\bar{\varepsilon}<<J S$ ( $J$ is the integral of $s-d$ exchange, $S$ is a magnetic ion spin).

This inequality makes it possible to confine our consideration to a subzone with $\sigma=\uparrow$, so that the spin index may be omitted. The effect of the high-frequency field on electron-magnon collisions can be observed by a method used in [7.8]. Moreover, we shall assume that the direct effect of the magnetic field of CLB on magnons is weak as compared with the electron-magnon interaction.

In the general case, the kinetic equations for electrons and magnons should be the quantum ones, and we shall derive them on the basis of quantum analogies of the microscopic distribution functions for electrons $\hat{f}$ and magnons $\hat{N}$ in the Wigner representation:

$$
\begin{aligned}
& \hat{f}(\vec{r}, \vec{G}, t)=\frac{1}{V} \sum_{\vec{k}} \exp \{-i \vec{k} \vec{r}\} a_{\vec{G}+\frac{\hbar \vec{k}}{2}}^{+} a_{\vec{G}-\frac{\hbar \vec{k}}{2}}, \\
& \hat{N}(\vec{r}, \vec{q}, t)=\frac{1}{V} \exp \{-i \vec{g} \vec{r}\} b_{\vec{q}+\frac{\hbar g}{2}}^{+} b_{\vec{q}-\frac{\hbar \vec{g}}{2}}
\end{aligned}
$$

where $V$ is the volume of FMSC, $\vec{G}=\vec{p}-(e / c) \vec{A}(\vec{r}, t)$ is the kinematic electron momentum.

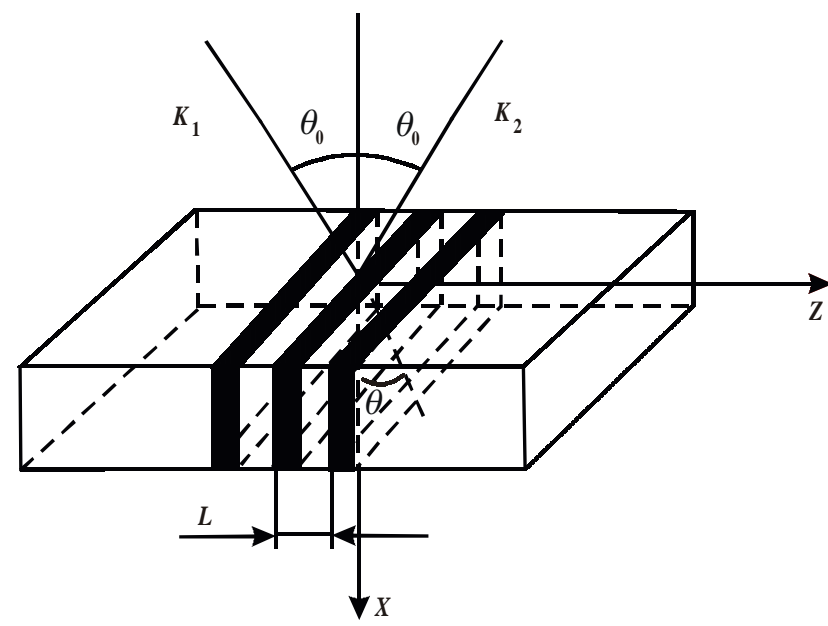

Fig.1. An interference picture created by two coherent light beams.
Then, from the motion equations for the operators $\hat{f}$ and $\hat{N}$ with the full Hamiltonian of electron-magnon system in the field of CLB we have

$$
i \hbar \frac{d \hat{f}}{d t}=i \hbar \frac{\partial \hat{f}}{\partial t}+[\hat{f}, \hat{H}] \quad i h \frac{d \hat{N}}{d t}=i \hbar \frac{\partial \hat{N}}{\partial t}+[\hat{N}, \hat{H}]
$$

after calculation of the commutations in (2) and averaging the products of these operators on density matrix of the electron-magnon system of FMSC, we obtained a set of quantum kinetic equations for ordinary distribution functions of electrons $f(\vec{r}, \vec{G}, t)$ and magnons $N(\vec{r}, \vec{q}, t)$. This set of quantum kinetic equations for electrons and magnons was obtained in $[3,4]$ and has the following form:

$$
\begin{aligned}
& \frac{\partial f}{\partial t}+\frac{\vec{G}}{m} \frac{\partial f}{\partial r}+e \vec{F}_{0} \frac{\partial f}{\partial \vec{G}}+ \\
& +\frac{e}{m c}\left\{\left[\left(\vec{G}-\frac{e}{c} \vec{A}\right) \nabla\right] \vec{A}+\left[\left(\vec{G}-\frac{e}{c} \vec{A}\right) \times \nabla(\nabla \times \vec{A})\right]\right\} \frac{\partial f}{\partial \vec{G}}= \\
& =\frac{1}{i \hbar} \sum_{\vec{q}, \vec{\eta}}|I(\vec{q})|^{2} \sum_{n, l=-\infty}^{+\infty} J_{n}\left(\frac{e \gamma_{\vec{\eta}}}{m \hbar \omega c}\right) J_{l}\left(\frac{e \gamma_{\vec{\eta}}}{m \hbar \omega c}\right) \times
\end{aligned}
$$$$
\times e^{i(n-l)\left(\omega t-X_{\vec{r}_{1}}\right)} \int_{-\infty}^{0} d t^{\prime} \times
$$$$
\times\left[f\left(\vec{r}, \vec{G}-\vec{r}_{1}, t+t^{\prime}\right)\left(1+f\left(\vec{r}, \vec{G}, t+t^{\prime}\right)\right) \times\right.
$$$$
\times N\left(\vec{r}, \vec{q}+\vec{r}_{1}, t^{\prime}+t\right)\left(1+N\left(\vec{r}, \vec{q}, t+t^{\prime}\right)\right)-
$$$$
-f\left(\vec{r}, \vec{G}, t+t^{\prime}\right)\left(1+f\left(\vec{r}, \vec{G}-\vec{r}_{1}, t+t^{\prime}\right)\right) \times
$$$$
\left.\times N\left(\vec{r}, \vec{q}, t+t^{\prime}\right)\left(1+N\left(\vec{r}, \vec{q}+\vec{r}_{1}, t+t^{\prime}\right)\right)\right] \times
$$$$
\exp \left[-\frac{1}{i \hbar}\left(\varepsilon_{\vec{G}-\vec{\eta}}-\varepsilon_{\vec{G}}+\omega_{\vec{q}+\vec{\eta}}-\omega_{\vec{q}^{\prime}}-l \hbar \omega+i \Delta\right) t^{\prime}\right]
$$$$
\frac{d N}{\partial t}+\frac{\partial \omega_{\vec{q}}}{\partial \vec{q}} \frac{\partial N}{\partial \vec{r}}=
$$$$
=\left.\frac{1}{\left(i \hbar^{2}\right)} \sum_{\vec{G}, \vec{r}} \sum_{l, l^{\prime}=-\infty}^{\infty} J_{l}\left(\frac{e \gamma_{\overrightarrow{\eta_{1}}}}{m c \hbar \omega}\right) J_{l^{\prime}}\left(\frac{e \gamma_{\overrightarrow{\eta_{1}}}}{m c \hbar \omega}\right) I_{e m}\right|^{2} \times
$$$$
\times \int_{-\infty}^{t} d t^{\prime}\left[f\left(\vec{G}-\vec{r}_{1}, \vec{r}, t^{\prime}\right)\left(1-f\left(\vec{G}, \vec{r}, t^{\prime}\right)\right) \times\right.
$$$$
\times N\left(\vec{q}+\vec{r}_{1}, \vec{r}, t^{\prime}\right)\left(1+N\left(\vec{q}, \vec{r}, t^{\prime}\right)\right)-
$$$$
-f\left(\vec{G}, \vec{r}, t^{\prime}\right)\left(1-f\left(\vec{G}-\overrightarrow{r_{1}}, \vec{r}, t^{\prime}\right)\right) \times
$$$$
\left.\times N\left(\vec{q}, \vec{r}, t^{\prime}\right)\left(1+N\left(\vec{q}+\vec{r}_{1}, \vec{r}, t^{\prime}\right)\right)\right] \times
$$$$
\times \exp \left\{-\frac{1}{i \hbar}\left(\left[\varepsilon_{\vec{G}-\vec{\eta}}-\varepsilon_{\vec{G}}+\omega_{\vec{q}+\vec{\eta}}-\omega_{\vec{q}}\right]\left(t-t^{\prime}\right)\right)\right\} \times
$$$$
\times e^{i \omega\left(l t-l^{\prime} t^{\prime}\right)} e^{i\left(l-l^{\prime}\right) X_{\vec{r}}}+
$$$$
+\frac{1}{\hbar^{2}} \sum_{k 123}\left|\Phi_{4 m p}\right|^{2} \delta_{2+3}^{\vec{k}+\vec{q}+1} \times
$$$$
\times \int_{-\infty}^{t} d t^{\prime}\left[(1+n(\vec{k}, t))\left(1+N\left(\vec{r}, \vec{q}, t^{\prime}\right)\right) \times\right.
$$$$
\times N\left(\vec{r}, 2, t^{\prime}\right) N\left(\vec{r}, 3, t^{\prime}\right)\left(1+N\left(\vec{r}, 1, t^{\prime}\right)\right)-
$$ 
O.Yu. Semchuk et al.: Features of optical properties of ferromagnetic ...

$-(n(\vec{k}, t))\left(N\left(\vec{r}, \vec{q}, t^{\prime}\right)\right) N\left(\vec{r}, 1, t^{\prime}\right) \times$

$\times\left(1+N\left(\vec{r}, 2, t^{\prime}\right)\left(1+N\left(\vec{r}, 3, t^{\prime}\right)\right)\right] \times$

$\times \exp \left[-\frac{1}{i \hbar}\left(\left(v_{\vec{r}}+\omega_{\vec{q}}+\omega_{1}-\omega_{2}-\omega_{3}\right)\right)\left(t-t^{\prime}\right)\right]$.

In this equation the following values are:

$J_{n}(x)$ is the Bessel function,

$X_{\vec{\eta}}=\operatorname{arctg}\left(\frac{\sum_{j} \vec{A}_{j} \vec{r}_{1} \sin \left(\vec{k}_{j} \vec{r}_{1}+\varphi_{j}\right)}{\sum_{j} \vec{A}_{j} \vec{r}_{1} \cos \left(\vec{k}_{j} \vec{r}_{1}+\varphi_{j}\right)}\right)^{\prime}$,
$\gamma_{\vec{\eta}}=\sum_{j j^{\prime}}\left(\vec{r}_{1} \vec{A}_{j}\right)\left(\vec{r}_{1} \vec{A}_{j^{\prime}}\right) \cos \left\{\left(\vec{k}_{j}-\vec{k}_{j^{\prime}}\right) \vec{r}+\varphi_{j}-\varphi_{j^{\prime}}\right\}$

It follows from the analysis of the equations (3) that the high frequency field of CLB acts on the FMSC electronmagnon system in two ways. Firstly, the electrons are affected an additional pressure caused by the light (the third term in the left part of Eq. (3)). Secondly, the integral of electron-magnon collisions becomes a periodic function of the coordinate $\vec{r}$. It is this periodic variation of the light pressure, along with the periodic dependence of collision integral on coordinate $\vec{r}$, that causes the formation in FMSC grating of carrier density, electric field intensity, electron and magnon temperatures, etc. For the first time, they were discussed in $[7,8]$. The electrical and optical properties of the FMSC with a static gratings have been recently [5-6, 9$10]$, but FMSC with a dynamical grating has not been investigated up to now.

\section{Optical properties of FMSC with a dynamic grating}

In this work we consider the dynamic grating- the grating coupling with the terms $s=1$ and $s=-1$ from the distribution functions of electrons $f(\vec{r}, \vec{G}, t)$ and magnons $N(\vec{r}, \vec{q}, t)$ time dependencies which can be written as

$$
\begin{aligned}
& f(\vec{r}, \vec{G}, t)=\sum_{s=-\infty}^{+\infty} e^{i s \omega t} f^{(s)}(\vec{r}, \vec{G}, t), \\
& N(\vec{r}, \vec{q}, t)=\sum_{m=-\infty}^{+\infty} e^{i m \omega t} N^{(m)}(\vec{r}, \vec{q}, t) .
\end{aligned}
$$

If the inequalities

$\hbar \omega>>T \gg \hbar v_{e m}, \omega>>P k / m, \omega>>F_{0} / P, \omega>>\tau^{-1}$

are valid, we may omit in the electron-magnon collision integrals all terms $f^{(s)}(\vec{r}, \vec{G}, t)$ and $N^{(m)}(\vec{r}, \vec{q}, t)$ with $s \neq 0, \quad m \neq 0$ and in the left part of kinetic equation for electrons (3) and magnons omit small terms and for the harmonics of the electron distribution function obtain the next equation

$$
\begin{aligned}
& \frac{\partial f(\vec{r}, \vec{G}, t)}{\partial t} \approx \frac{2 \pi}{\hbar} \sum_{\vec{q}, \vec{\eta}}\left|I_{e m}(\vec{q})\right|^{2} \\
& \sum_{s, l=-\infty}^{\infty} J_{s+l}\left(\frac{e \gamma\left(r_{1}\right)}{m c \omega}\right) J_{l}\left(\frac{e \gamma\left(\vec{r}_{1}\right)}{m c \omega}\right) \times \\
& \times e^{i s\left(\omega t-X_{\vec{r}}\right)}\left[f^{(0)}\left(\vec{r}, \vec{G}-\vec{r}_{1}\right) N^{(0)}(\vec{r}, \vec{q}+\vec{r}) \times\right. \\
& \times\left(1+N^{(0)}(\vec{r}, \vec{q})\right)-f^{(0)}(\vec{r}, \vec{G}) N^{(0)}(\vec{r}, \vec{q}) \times \\
& \left.\times\left(1+N^{(0)}(\vec{r}, \vec{q}+\vec{r})\right)\right] \times \\
& \times \delta\left(\varepsilon_{\vec{G}-\vec{\eta}}-\varepsilon_{\vec{G}}+\omega_{\vec{q}+\vec{\eta}}-\omega_{\vec{q}}-l \hbar \omega\right)
\end{aligned}
$$

Now we consider the left part of the equation (5). To content the term of $s=+1$ at the series of (4) we obtained (we suppose that $f^{( \pm 1)}(\vec{r}, \vec{G}, t) \approx f^{( \pm 1)}(\vec{r}, \vec{G})$ as these amplitudes are very slowly changing functions of time $t \sim \omega^{-1}$ )

$$
\frac{\partial f(\vec{r}, \vec{G}, t)}{\partial t} \approx i \omega e^{i \omega t} f^{(+1)}(\vec{r}, \vec{G})
$$

Similar calculation for the term with $s=-1$ gives

$$
\frac{\partial f(\vec{r}, \vec{G}, t)}{\partial t} \approx-i \omega e^{-i \omega t} f^{(-1)}(\vec{r}, \vec{G}) .
$$

Using $f^{(0)}(\vec{r}, G)<<1$ (this takes place at the moderate electron concentrations) and $N^{(0)}(\vec{r}, \vec{q})>>1$ (this take place at the moderately low temperatures) as well as put successive (7) and (8) to (5) we obtain

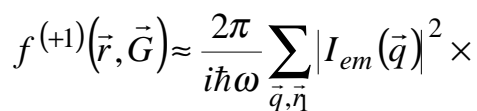

$$
\begin{aligned}
& \times \sum_{l=-\infty}^{+\infty} J_{l}\left(\frac{e \gamma\left(\vec{r}_{1}\right)}{m c \omega}\right) J_{l+1}\left(\frac{e \gamma\left(\vec{r}_{1}\right)}{m c \omega}\right) e^{-i X_{\overrightarrow{1}}} \times \\
& \times N^{(0)}(\vec{r}, \vec{q}) N^{(0)}\left(\vec{r}, \vec{q}+\vec{r}_{1}\right) \times \\
& \times\left\{f^{(0)}\left(\vec{r}, \vec{G}-\vec{r}_{1}\right)-f^{(0)}(\vec{r}, \vec{G})\right\} \times \\
& \times \delta\left(\varepsilon_{\vec{G}-\vec{\eta}}-\varepsilon_{\vec{G}}+\omega_{\vec{q}+\vec{\eta}}-\omega_{\vec{q}}-l \hbar \omega\right)
\end{aligned}
$$

$$
f^{(-1)}(\vec{r}, \vec{G}) \approx-\frac{2 \pi}{i \hbar \omega} \sum_{\vec{q}, \vec{\eta}}\left|I_{e m}(\vec{q})\right|^{2} \times
$$

$\times \sum_{l=-\infty}^{+\infty} J_{l}\left(\frac{e \gamma\left(\vec{r}_{1}\right)}{m c \omega}\right) J_{l-1}\left(\frac{e \gamma\left(\vec{r}_{1}\right)}{m c \omega}\right) e^{i X_{\overrightarrow{1}}} \times$

$\times N^{(0)}(\vec{r}, \vec{q}) N^{(0)}\left(\vec{r}, \vec{q}+\vec{r}_{1}\right) \times$

$\times\left\{f^{(0)}\left(\vec{r}, \vec{G}-\vec{r}_{1}\right)-f^{(0)}(\vec{r}, \vec{G})\right\} \times$

$\times \delta\left(\varepsilon_{\vec{G}-\vec{\eta}}-\varepsilon_{\vec{G}}+\omega_{\vec{q}+\vec{\eta}}-\omega_{\vec{q}}-l \hbar \omega\right)$. 
When we have the expression for the harmonics of the electron distribution function, we can investigate the dynamical processes in FMSC. For this purpose, let the function be given as

$$
\tilde{f}_{1}(\vec{r}, \vec{G}, t)=e^{i \omega t} f^{(+1)}(\vec{r}, \vec{G})+e^{-i \omega t} f^{(-1)}(\vec{r}, \vec{G}) .
$$

After substitution (9) and (10) to the (11) and limiting the only one- and two-photon processes $(l=1,2)$ the next expression for the HF-part of first harmonics of the electron distribution function (11) can be obtained

$$
\begin{aligned}
& \tilde{f}_{1}(\vec{r}, \vec{G}, t) \approx \frac{2 \pi}{i \hbar \omega} \sum_{\vec{q}, \vec{n}}\left|I_{e m}(\vec{q})\right|^{2} N^{(0)}(\vec{r}, \vec{q}) \times \\
& \times N^{(0)}\left(\vec{r}, \vec{q}+\vec{r}_{1}\right)\left\{f^{(0)}\left(\vec{r}, \vec{G}-\vec{r}_{1}\right)-f^{(0)}(\vec{r}, \vec{G})\right\} \times \\
& \times\left(e^{i\left(\omega t-X_{\vec{\eta}}\right)}-e^{-i\left(\omega t-X_{\vec{\eta}}\right)}\right) \times \\
& \times \sum_{l=1}^{2} J_{1}\left(\frac{e \gamma_{\vec{\eta}}}{m c \hbar \omega}\right) \times \\
& \times\left[J_{2}\left(\frac{e \gamma_{\vec{\eta}}}{m c \hbar \omega}\right)+(2-l) J_{l-1}\left(\frac{e \gamma_{\vec{\eta}}}{m c \hbar \omega}\right)\right] \times \\
& \times\left[\delta\left(\varepsilon_{\vec{G}-\vec{\eta}}-\varepsilon_{\vec{G}}+\omega_{\vec{q}+\vec{\eta}-} \omega_{\vec{q}}-l \hbar \omega\right)_{-}\right. \\
& \left.-\delta\left(\varepsilon_{\vec{G}-\vec{\eta}}-\varepsilon_{\vec{G}}+\omega_{\vec{q}+\vec{\eta}-} \omega_{\vec{q}}+l \hbar \omega\right)\right]
\end{aligned}
$$

Using a well known equation

$$
e^{i X}-e^{-i X}=2 i \sin X \text {, }
$$

we may rewrite (11) as the following

$$
\begin{aligned}
& \tilde{f}_{1}(\vec{r}, \vec{G}, t) \approx \frac{2 \pi}{\hbar \omega} \sum_{\vec{q}, \vec{r}}\left|I_{e m}(\vec{q})\right|^{2} N^{(0)}(\vec{r}, \vec{q}) \times \\
& \times N^{(0)}\left(\vec{r}, \vec{q}+\vec{r}_{1}\right)\left\{f^{(0)}\left(\vec{r}, \vec{G}-\vec{r}_{1}\right)-f^{(0)}(\vec{r}, \vec{G})\right\} \times \\
& \times \sum_{l=1}^{2} J_{1}\left(\frac{e \gamma_{\vec{\eta}}}{m c \hbar \omega}\right) \times \\
& \times\left[J_{2}\left(\frac{e \gamma_{\vec{n}}}{m c \hbar \omega}\right)+(2-l) J_{l-1}\left(\frac{e \gamma_{\vec{\eta}}}{m c \hbar \omega}\right)\right] \times \\
& \times \sin \left(\omega t-X_{\vec{\eta}}\right) \times \\
& \times\left[\delta\left(\varepsilon_{\vec{G}-\vec{\eta}}-\varepsilon_{\vec{G}}+\omega_{\vec{q}+\vec{\eta}}-\omega_{\vec{q}}-l \hbar \omega\right)-\right. \\
& \left.-\delta\left(\varepsilon_{\vec{G}-\vec{\eta}}-\varepsilon_{\vec{G}}+\omega_{\vec{q}+\vec{\eta}}-\omega_{\vec{q}}+l \hbar \omega\right)\right) .
\end{aligned}
$$

Now we calculate the HF-current, which is determined

$$
\vec{j}(\vec{r}, t)=-\frac{e^{2} n(\vec{r})}{m c} \vec{A}(\vec{r}, t)+\frac{e}{m} \int \vec{G} f(\vec{r}, \vec{G}, t) d \vec{G}
$$

In the linear approximation for $\vec{A}_{j}$ the HF-current can be expressed as

$$
\vec{j}_{1}(\vec{r}, t)=-\frac{e^{2} n(\vec{r})}{m c} \vec{A}(\vec{r}, t)+\frac{e}{m} \int \vec{G} \tilde{f}_{1}(\vec{r}, \vec{G}, t) d \vec{G} .
$$

Substituting (14) into (13)), limiting by the only onephoton processes, assuming that $\vec{A}_{j} \perp \vec{F}_{0}$ and under influence on the FMSC surface of two waves with $\vec{A}_{1} \mid \vec{A}_{2}$, when there are two symmetrically oriented beams with $k_{1 x}=k_{2 x}, \quad k_{1 z}=-k_{2 z}$, we obtain

$$
\begin{aligned}
& \vec{j}_{1}(\vec{r}, \pm \omega)=-\frac{e^{2} n(\vec{r})}{2 m c}\left[1-\frac{i}{3 \omega \tau(\hbar \omega)}\right] \times \\
& \times \sum_{j} \vec{A}_{j} \exp \left\{ \pm i\left(\vec{k}_{j} \vec{r}+\varphi_{j}\right)\right\}
\end{aligned}
$$

where

$$
\tau(\hbar \omega)=\frac{8 \pi^{2} \hbar^{7}}{a^{6} m m_{s}^{2} T^{2}} \sqrt{\frac{J S}{\hbar \omega}} .
$$

Thus, the dependence of $\vec{j}_{j}$ on the coordinate $r$ can be used to determine the dynamic grating in FMSC crystal.

This grating coupling with the electromagnetic wave absorption coefficient of FMSC crystal $\alpha_{e}$ is determined by the HF-current and can be written as [11]

$\tau(\hbar \omega)=\frac{8 \pi^{2} \hbar^{7}}{a^{6} m m_{s}^{2} T^{2}} \sqrt{\frac{J S}{\hbar \omega}}$,

where $\varepsilon_{0}$ and $\mu_{0}$ are the dielectric and magnetic susceptibilities of the FMSC crystal, respectively; $A_{0}$ is average on the period of CLB of the value $\vec{A} ;<\ldots \ldots>$ is the averaging on the period of CLB.

The second term of the approximations of the HFcurrent $\vec{j}_{3}( \pm \omega)$ is proportional to $\vec{A}_{j}^{3}$ and its components on the axis $j_{3 i}( \pm \omega)$ can be written as

$$
\begin{aligned}
& j_{3 i}(\vec{r}, \pm \omega)=-i \frac{n(\vec{r}) c}{60 \hbar \omega \tau(\hbar \omega) m^{2} \omega}\left(\frac{e}{c}\right)^{2} \times \\
& \times\left[1-\frac{2 \tau(\hbar \omega)}{\tau(2 \hbar \omega)}\right] \sum_{j} \exp \left[ \pm\left(\vec{k}_{j} \vec{r}-\varphi_{j}\right)\right] \times \\
& \sum_{j^{\prime}, j^{\prime \prime}}\left[\left(A_{j i}\right)\left(\vec{A}_{j^{\prime}} \vec{A}_{j^{\prime \prime}}\right)+A_{j^{\prime} j}\left(\vec{A}_{j} \vec{A}_{j^{\prime \prime}}\right)+A_{j^{\prime \prime} j}\left(\vec{A}_{j} \vec{A}_{j^{\prime}}\right)\right] \times \\
& \times \cos \left[\left(\vec{k}_{j^{\prime}}-\vec{k}_{j^{\prime \prime}}\right) \vec{r}+\varphi_{j^{\prime}}-\varphi_{j^{\prime \prime}}\right]
\end{aligned}
$$
as 


\section{O.Yu. Semchuk et al.: Features of optical properties of ferromagnetic ...}

This current is responsible for the nonlinear absorption of the energy of the electromagnetic wave on the fundamental harmonic of the electron distribution function. At $\vec{A}_{j} \perp \vec{F}_{0}$, in the case when only two symmetrically oriented CLB with $\vec{A}_{1} \| \vec{A}_{2}, k_{1 x}=k_{2 x}, k_{1 z}=-k_{2 z}$, the expression for $j_{3}(\vec{r}, \pm \omega)$ is very simple and the coefficient $\alpha_{e}$ can be written in the form:

$$
\begin{aligned}
& \alpha_{e}=\frac{4 \pi n_{0} e^{2}}{3 m c \omega^{2} \tau(\hbar \omega) \sqrt{\varepsilon_{0} \mu_{0}}} \times \\
& \times\left\{1-\frac{3 e^{2} A_{1} A_{2}}{10 m c^{2} \hbar \omega} \times\right. \\
& \left.\times\left[\frac{A_{1}}{A_{2}}+\frac{A_{2}}{A_{1}}+2 \cos \left(2 k_{1 z} z+\varphi_{1}-\varphi_{2}\right)\right]\right\} .
\end{aligned}
$$

The first term in (17) is associated with the electromagnetic wave absorption coefficient of free electrons in the quantum frequency range, and the second term is the nonlinear response of the absorption coefficient of weakly wave. The peculiarity of the second term is the dependence on coordinate $z$, which rises from the dependence of the intensity of the electromagnetic wave passed through a FMSC crystal. Thus, the presence of the dynamic grating leads to the modulation of the light absorption coefficient of FMSC crystal that can be detected by the changes of the outgoing intensity of the sensing wave passed through FMSC crystal. This result can be illustrated like that. Let weakly electromagnetic wave

$$
\vec{E}(z, t)=\vec{E}_{0}(z) \exp \left[i\left(\omega_{0} t-k_{0} z\right)\right]
$$

propagate in FMSC. Its amplitude $\vec{E}_{d}$ after passing through the FMSC crystal with thickness $d$ is as following

$$
\vec{E}_{d}=\vec{E}_{0} \exp \left\{-\alpha_{e} d / 2\right\} \exp \left\{i \frac{2 \pi e^{2} n_{1}(z) d}{\sqrt{\varepsilon_{0} \mu_{0}} m c \omega_{0}}\right\} .
$$

As it follows from the (19), the parameters of the dynamic grating in FMSC are to be determined by the changes of the outgoing intensity of the sensing wave (18) passed through the FMSC crystal with a thickness $d$.

Now we shall investigate frequency dependence of absorption coefficient. In Fig.2, the frequency dependence of absorption coefficient is plotted.

Fig. 2 shows that with increasing frequency, the nonlinear absorption coefficient grows, reaching a maximum at the frequency $\omega \approx 3 \cdot 10^{12} \mathrm{~s}^{-1}$, and further smoothly decreases. This result can explain the influence of the dynamic grating of electron concentration on the processes of propagation and absorption of electromagnetic waves in FMSC.

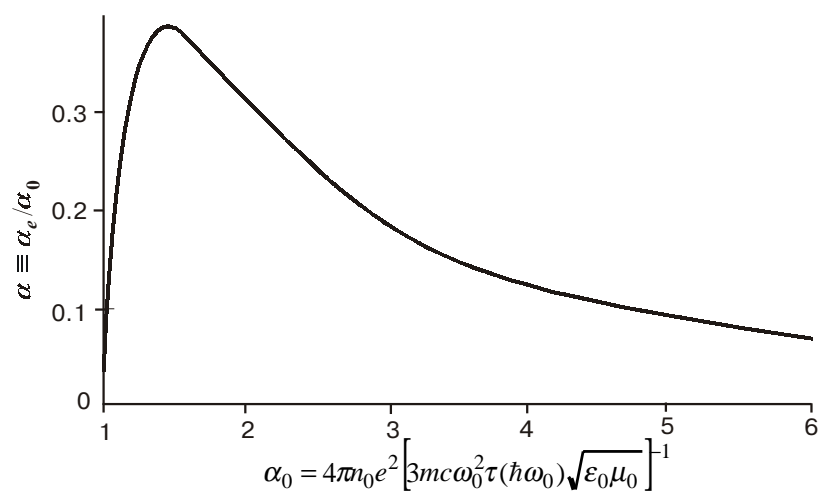

Fig.2. Frequency dependence of light absorption coefficient $\left(x=\omega / \omega_{0}, \omega_{0}=2 \cdot 10^{12} s^{-1}\right)$.

\section{Conclusions}

As it follows from our discussion, in FMSC coherent light beams produce a dynamic grating, which is determined by high-frequency current and described by the high-frequency harmonics of the electrons distribution functions. This dynamic grating couples with the light (infrared) absorption coefficient of FMSC crystal. This coefficient consists of two terms. The first term is the infrared light absorption coefficient of free electrons in the quantum frequency range. The peculiarity of the second term is the dependence on coordinate $z$, that can be manifestation of the dependence of electromagnetic wave intensity, transmitted through a FMSC crystal. The presence of the dynamic grating of electron concentration essentially changes optical properties of FMSC crystals. For example, at the increasing of weakly wave frequency, the nonlinear absorption coefficient grows, reaching a maximum at frequency $\omega \approx 3 \cdot 10^{12} \mathrm{~s}^{-1}$, and further smoothly decreases with higher frequencies.

\section{References}

1. V.P. Seminozhenko, Kinetics of interacting quasiparticles in strong external fields // Phys. Rep., 91 (3), p.103-182 (1983).

2. O.Yu. Semchuk, A.E Levshin and P.M. Tomchuk // Sov.Phys. Solid State, 28 (2), p.229-232 (1986).

3. O.Yu. Semchuk, L.G. Grechko and V.M. Ogenko, Field influence on the parameters of ferromagnetic semiconductor superlattices formed by coherent light beams // Phys.stat.sol. (b), 157 (1), p.451-58 (1990).

4. O.Yu. Semchuk, L.G. Grechko and V.M. Ogenko, Electrons and magnons of a ferromagnetic semicondcutors in the field of coherent light beams // Ukr.Phys.Jour., 38 (7), p.982-1001 (1993).

5. O.Yu. Semchuk, L.G. Grechko and V.M. Ogenko, A new method of the optical diagnostics of materials for opto-, micro- and quantum electronics on the base of the semiconductor superlattices //Proc. SPIE, 2648, p.48 (1996).

6. O.Yu. Semchuk, The peculiarity of the volt-current characteristic of the ferromagnetic semiconductor with superlattice on nonequilibrium electrons and magnons // Ukr.Phys.Jour., 42 (11-12), p.1369 (1997). 
O.Yu. Semchuk et al.: Features of optical properties of ferromagnetic ...

7. A.I. Akhiezer, B.G. Baryakhtar and S.B. Peletminskii, Spin Waves. Moscow, Nauka, 1967.

8. O.Yu. Semchuk, and S.S. Rozhkov, Transient magnetic susceptibility of ferromagnetic semiconductors // Sov. Phys. Solid State, 24 (10), p.1730-1732 (1982).

9. O.Yu. Semchuk, L.G. Grechko and V.M. Ogenko, Theory of transport phenomena in ferromagnetic semiconductors with superlattice produced by coherent light beams // Physica B, 176, p.153-155 (1992)

10. O.Yu. Semchuk, L.G. Grechko, V.M. Ogenko, Theory of transport phenomena in ferromagnetic semiconductors with superlattice produced by coherent light beams // Phys.stat.sol. (b), 162, p.539-543 (1990).

11. X. Fen, Photon-electron interaction in crystals at the absence of external fields. Moscow, Mir, 1969, 127p. 